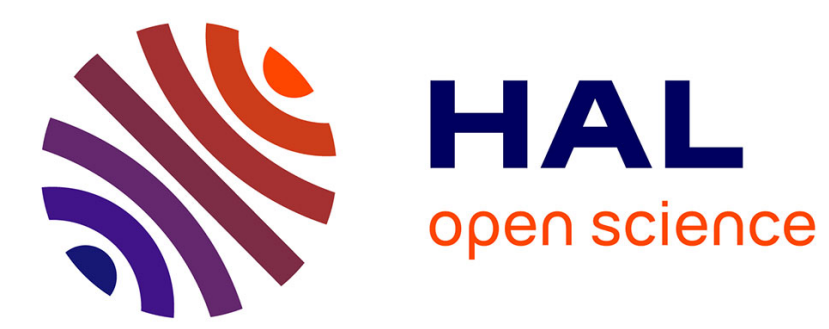

\title{
Did a large impact reorient the Moon?
}

Mark A. Wieczorek, Mathieu Le Feuvre

\section{- To cite this version:}

Mark A. Wieczorek, Mathieu Le Feuvre. Did a large impact reorient the Moon?. Icarus, 2009, 200

(2), pp.358. 10.1016/j.icarus.2008.12.017 . hal-00517248

\section{HAL Id: hal-00517248 \\ https://hal.science/hal-00517248}

Submitted on 14 Sep 2010

HAL is a multi-disciplinary open access archive for the deposit and dissemination of scientific research documents, whether they are published or not. The documents may come from teaching and research institutions in France or abroad, or from public or private research centers.
L'archive ouverte pluridisciplinaire HAL, est destinée au dépôt et à la diffusion de documents scientifiques de niveau recherche, publiés ou non, émanant des établissements d'enseignement et de recherche français ou étrangers, des laboratoires publics ou privés. 


\section{Accepted Manuscript}

Did a large impact reorient the Moon?

Mark A. Wieczorek, Mathieu Le Feuvre

PII:

S0019-1035(08)00428-4

DOI: $\quad$ 10.1016/j.icarus.2008.12.017

Reference: $\quad$ YICAR 8838

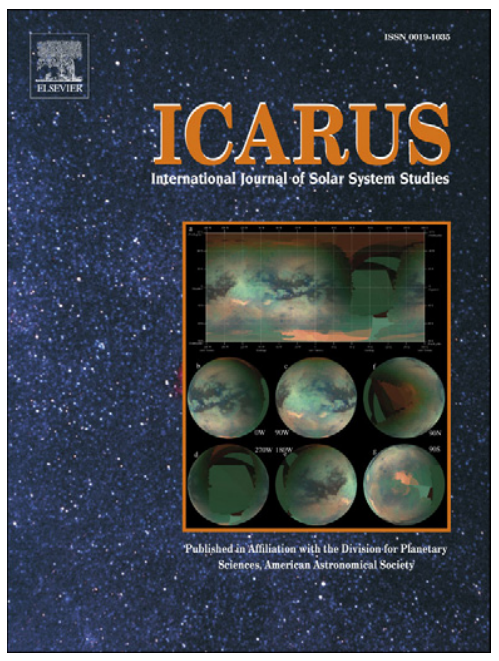

To appear in: Icarus

Received date: 19 September 2008

Revised date: 10 December 2008

Accepted date: 15 December 2008

Please cite this article as: M.A. Wieczorek, M. Le Feuvre, Did a large impact reorient the Moon?, Icarus (2009), doi: 10.1016/j.icarus.2008.12.017

This is a PDF file of an unedited manuscript that has been accepted for publication. As a service to our customers we are providing this early version of the manuscript. The manuscript will undergo copyediting, typesetting, and review of the resulting proof before it is published in its final form. Please note that during the production process errors may be discovered which could affect the content, and all legal disclaimers that apply to the journal pertain. 


\title{
Did a large impact reorient the Moon?
}

\author{
Mark A. Wieczorek and Mathieu Le Feuvre \\ Institut de Physique du Globe de Paris, Saint Maur des Fossés, France \\ Copyright (c) 2008 Mark A. Wieczorek
}

\begin{abstract}
The Moon is currently locked in a spin-orbit resonance of synchronous rotation, of which one consequence is that more impacts should occur near the Moon's apex of motion $\left(0^{\circ} \mathrm{N}, 90^{\circ} \mathrm{W}\right)$ than near its antapex of motion $\left(0^{\circ} \mathrm{N}, 90^{\circ} \mathrm{E}\right)$. Several of the largest lunar impact basins could have temporarily unlocked the Moon from synchronous rotation, and after the re-establishment of this state the Moon would have been left in either its initial orientation, or one that was rotated $180^{\circ}$ about its spin axis. We show that there is less than a $2 \%$ probability that the oldest lunar impact basins are randomly distributed across the lunar surface. Furthermore, these basins are preferentially located near the Moon's antapex of motion, and this configuration has less than a $0.3 \%$ probability of occurring by chance. We postulate that the current "near side" of the Moon was in fact its "far side" when the oldest basins formed. One basin with the required size and temporal characteristics to account for a $180^{\circ}$ reorientation is the Smythii basin.
\end{abstract}

Key words: Moon, cratering, rotational dynamics

\section{Introduction}

The Moon is the type example of a synchronously locked natural satellitefor every orbit it makes about the Earth, it rotates once about its spin axis such that the same hemisphere is always directed towards the Earth. One consequence of this spin-orbit resonance is that the Moon should be subjected to a higher impact flux on its western hemisphere than its eastern hemisphere (e.g., Wood, 1973; Shoemaker and Wolf, 1982; Horedt and Neukum, 1984). Another ramification is that the net quantity of solar wind-implanted volatiles in the lunar regolith should be larger on the lunar far side than its near side as a result of the Earth's protective magnetosphere (e.g., Johnson et al., 1999). Furthermore, the tidal potential of the Earth would have given rise to a static tidal bulge on the Moon that could have been frozen into its lithosphere shortly 
after lunar formation (e.g., Lambeck and Pullan, 1980; Garrick-Bethell et al., 2006).

The synchronous rotation of the Moon is a direct consequence of this body's irregular gravitational field and tides raised by the Earth. If the Moon was once spinning faster or slower than synchronous, gravitational torques and internal energy dissipation would have acted to either decrease or increase its rotation rate, respectively (e.g., Burns, 1986). Ultimately, on the time frame of a thousand years, the minimum potential and kinetic energy configuration of the Moon would have been achieved, where its minimum moment of inertia is directed along the Earth-Moon axis and where its maximum moment of inertia is aligned with its spin axis. Since the moments of inertia of a body are unchanged by a $180^{\circ}$ rotation about any of its three principal axes, there are currently two equally probable equilibrium orientations for the Moon.

When asteroids and comets collide with the lunar surface, the angular momentum and spin rate of the Moon are altered instantaneously. Since the relative change in angular momentum is small, the Moon will continue to rotate approximately about its prior spin axis, but with the axis of its minimum moment of inertia librating back and forth in longitude like a pendulum (e.g., Melosh, 1975; Peale, 1975). The maximum angular extent of these librations depends upon the change in angular momentum along the lunar rotation axis, and if the impulse delivered by the impactor were large enough, the libration amplitude could exceed $90^{\circ}$. If this were to happen, the Moon would rotate non-synchronously, allowing both faces to be seen from the surface of the Earth over a period of about a year. At this point, tidal torques would act to once again bring the Moon into synchronous rotation, but it would be only a matter of chance as to whether the same face of the Moon would be directed towards the Earth as before the impact (e.g., Melosh, 1975; Lissauer, 1985). The only impact events that could have unlocked the Moon from synchronous rotation are those that formed the giant impact basins, of which the youngest is about 3.8 billion years old (e.g., Stöffler and Ryder, 2001). If a reorientation of the Moon ever occurred, this event would have taken place before most of the visible mare basalts erupted and when the Moon was somewhat closer to the Earth.

We test the hypothesis that one or more impact events led to a $180^{\circ}$ reorientation of the Moon by use of the fact that a synchronously rotating satellite will be subjected to a higher impact flux on its western (leading) hemisphere than on its eastern (trailing) hemisphere. This asymmetry is a simple consequence of the different relative velocities that occur when the lunar surface is moving towards or away from an approaching bolide, and several studies have attempted to describe the expected spatial variations in the cratering rate under various simplifying assumptions (e.g., Wood, 1973; Shoemaker and Wolf, 1982; Horedt and Neukum, 1984; Zahnle et al., 2001). Two recent studies have 
quantified this effect by combining the impact probabilities of the near-Earth objects (i.e., those asteroids and comets that are capable of impacting the Earth and Moon) with direct orbital simulations to determine the impact locations on the lunar surface (Gallant et al., 2006; Le Feuvre, 2008). For the current population of near-Earth objects, the impact rate at the Moon's apex of motion is predicted to be greater by about $29 \%$ than at its antapex of motion (the simplified model of Zahnle et al. (2001) predicts a similar value), and this is consistent with the distribution of young rayed craters as measured by Morota and Furumoto (2003) and Morota et al. (2005). Since the magnitude of the apex-antapex asymmetry depends upon the Moon's orbital velocity, the spatial variations in the cratering rate would have been greater in the past when the Moon was closer to the Earth.

Given a list of impact craters ordered by relative age, we can determine the direction of the maximum cratering rate for various age groupings, and compare this to the Moon's current apex of motion. If the maximum cratering rate were found to be directed towards the Moon's antapex of motion (contrary to what might be expected), this would suggest that these craters could have formed when the Moon was in an orientation rotated $180^{\circ}$ with respect to its present configuration. If such a reorientation ever occurred, this would have important implications for the dating of surfaces by the crater chronology method as the expected spatial variations in the cratering rate before this event would be offset in longitude by $180^{\circ}$. The rotational energy dissipated in such an event could perhaps have affected temporarily the energetics and dynamics of the lunar core, and rotation of the Moon through the Earth's tidal potential could have possibly led to a global fracture system.

In this paper, we first re-examine the impact conditions that are required to spin up the Moon to a non-synchronous state. Following this, we investigate the distribution of lunar impact basins as a function of age and show that the oldest basins most likely formed when the current "near side" of the Moon was facing away from the Earth. We then discuss some of the implications of this result, and finally conclude by mentioning how future data sets could be used to refine this hypothesis.

\section{Reorientation of the Moon}

When objects collide with the Moon, the lunar spin rate and angular momentum are altered instantaneously. If the entire momentum of the projectile is transferred to the target, the magnitude of the change in angular rotation rate about the spin axis (here assumed to coincide with the axis of the largest 
principal moment of inertia $C$ ) is

$$
|\Delta \omega|=m v R \cos i|\cos \psi| / C,
$$

where $m$ is the mass of the bolide, $v$ is the impact velocity, $R$ is the radius of the Moon, $i$ is the impact angle measured with respect to the surface, and $\psi$ is the angle between the angular momentum of the bolide at the time of impact and the lunar spin axis. While the gravitational escape of slow moving impact ejecta would act to increase this estimate (Ahrens and Harris, 1994; Melosh et al., 1994; Holsapple, 2004), the enhancement from this phenomenon would be small for the Moon given this body's relatively high escape velocity.

Eq. 1 shows that the maximum change in the spin rate occurs when (a) the impact velocity is tangential to the surface, (b) the impact velocity is perpendicular to the spin axis, and (c) the impact occurs at the equator. (We note that impact craters are predicted to be circular for all $i$ greater than about $10^{\circ}$; see Bottke et al. (2000) and Herrick and Forsberg-Taylor (2003).) In order to put the Moon into non-synchronous rotation, the resulting libration angle in longitude must be greater than $90^{\circ}$, and this occurs when Eq. 1 is greater than the critical value (see Lissauer (1985) and Eq. 5 of Goldreich and Peale (1966))

$$
\omega_{0}=\Omega \sqrt{3\left(\frac{B-A}{C}\right) H(1, e)},
$$

where $\Omega$ is the orbital angular velocity of the Moon, $A<B<C$ are the three principal moments of inertia of the Moon, $e$ is the orbital eccentricity of the Moon, and

$$
H(1, e) \simeq 1-5 e^{2} / 2+13 e^{4} / 16
$$

For the current eccentricity of the lunar orbit, $0.055, H$ is approximately equal to 1 .

Using Eqs 1 and 2, the minimum-sized bolide required to disrupt the Moon's synchronous rotation can be determined. For these calculations, an average impact geometry was assumed by setting the two angles in Eq. 1 equal to their average value of $45^{\circ}$. Results are shown in the upper panel of Figure 1 as a function of impact velocity for two representative densities of cometary and asteroidal materials (500 and $8000 \mathrm{~kg} \mathrm{~m}^{-3}$ ) and for two different orbital velocities when the Moon was separated from the Earth by 25 and 50 Earth radii. We note that if the Moon formed near the Roche limit (at approximately 3 Earth radii), its semimajor axis would have increased rapidly over about a hundred million years to more than 25-35 Earth radii, after which time its 
semimajor axis would have increased with a slower rate to its actual value of 60 Earth radii (e.g., Webb, 1982; Ross and Schubert, 1989; Williams, 2000, 2004). The impact velocities plotted in this figure (from about 2 to $50 \mathrm{~km}$ $\mathrm{s}^{-1}$ ) span nearly the entire range of values that are possible for the current population of near-Earth objects (Le Feuvre and Wieczorek, 2008). For the present-day average impact velocity of about $19 \mathrm{~km} \mathrm{~s}^{-1}$, an object would have to be greater than about $50 \mathrm{~km}$ in order to unlock the Moon from synchronous rotation.

[Fig. 1 about here.]

Given the diameter, velocity and density of a bolide that collides with the Moon, the size of the corresponding crater on the lunar surface can be estimated using standard impact scaling laws (for a review, see Holsapple, 1993). For this calculation, it was assumed that only the vertical component of the impact velocity contributes to the final crater size, and the transient crater diameter was estimated using the scaling equations and constants appropriate for the gravity regime as summarized by Holsapple and Housen (2007). Gravitational collapse of the transient crater will give rise to a final crater rim diameter that is somewhat larger than predicted by these equations (e.g., Croft, 1985). Nevertheless, the relative importance of this enhancement is likely to be considerably smaller for impact basins than for complex craters (see discussion in Wieczorek and Phillips, 1999), and we will assume that the transient cavity and final rim diameter are approximately equal. When comparing theoretical crater sizes to actual lunar impact basins, the biggest uncertainty is in determining which ring of a multiring basin corresponds to the structure formed by the collapse of the transient crater (Wieczorek and Phillips, 1999).

The minimum crater diameter required to unlock the Moon from synchronous rotation is plotted in the lower panel of Figure 1 as a function of impact velocity for the case of an average impact geometry. These results demonstrate that while the impact velocity and bolide density both play a role in determining the minimum-sized crater diameter, the Earth-Moon separation has a much greater effect. For the case where the semimajor axis of the Moon is close to its present value, any impact crater greater than about $300 \mathrm{~km}$ in diameter could have unlocked the Moon from synchronous rotation. In contrast, if the semimajor axis of the Moon was closer to 25 Earth radii (which is most likely a lower bound when the basins formed), the crater diameter would have to be greater than about $350 \mathrm{~km}$ for the minimum impact velocity, and greater than about $500 \mathrm{~km}$ for the average impact velocity.

As originally noted by Melosh (1975), there are several impact basins that could have temporarily unlocked the Moon from synchronous rotation and which might have led ultimately to a $180^{\circ}$ reorientation about the lunar spin 
axis. Table 1 lists the known lunar impact basins along with their estimated sizes based on both photogeological and geophysical methods. Since many lunar impact basins possess multiple rings, it is not always evident from geologic considerations alone which of these rings corresponds to the structure formed by the collapse of the transient crater. In contrast, gravitational data are sensitive to subsurface density contrasts, and when combined with surface topography, it is possible to invert for lateral variations in crustal thickness. From the crustal thickness maps, it is then possible to estimate the size of the crater's excavation cavity, which is expected to be a close approximation to the initial transient crater diameter (Wieczorek and Phillips, 1999; Hikida and Wieczorek, 2007).

[Table 1 about here.]

The geophysical approach of estimating the size of a basin's excavation cavity has been applied successfully to only a dozen of the younger near-side impact basins, and these results demonstrate that the "main rim," as determined from photogeologic studies, is not always a good approximation to the transient crater diameter. Thus, in order to determine which of the basins in Table 1 could have unlocked the Moon from synchronous rotation, we consider only those whose diameters have been estimated by geophysical means. If we choose $500 \mathrm{~km}$ as the minimum crater diameter (which is appropriate for an EarthMoon separation of 25 Earth radii along with average impact conditions), we are left with four candidate craters: Imbrium, Serenitatis, Crisium, and Smythii. Of course, it is quite probable that some of the basins that lack geophysical-based diameter estimates are larger than $500 \mathrm{~km}$. It should also be noted that if the impact conditions were optimal (that is, the impact occurred at the equator, and the impact velocity was tangential to the surface and perpendicular to the spin axis), the minimum-sized crater necessary to unlock the Moon's synchronous rotation would be smaller by a factor of about 2. Even with this uncertainty, though, it should be noted that there are no craters younger than Orientale that are big enough to have unlocked the Moon from synchronous rotation.

\section{Evidence for reorientation}

One of the consequences of synchronous rotation is that more impact events should occur near the Moon's apex of motion on its western hemisphere than the antapex of motion on its eastern hemisphere. At the present time, the difference in impact rate between these two points is about $29 \%$, but this would have been greater in the past when the Moon was closer to the Earth and its orbital velocity was higher (see Le Feuvre, 2008; Zahnle et al., 2001). If one or more impact events unlocked the Moon from synchronous rotation, and if 
the geographic locations of the apex and antapex of motion were subsequently switched, one might expect to find a surplus of craters within a certain age range located near the current antapex of motion on the eastern hemisphere of the Moon. (If the population of impactors in the distant past were in geocentric orbits, as opposed to heliocentric orbits, asymmetries in the cratering rate might still exist, though the dependence in longitude would probably be different (Pinet, 1985).)

In order to test the hypothesis that the Moon has been reoriented about its spin axis by one or more impact events, we investigate the spatial distribution of ancient lunar impact basins. For this purpose, we make use of the known impact basins as tabulated by Wilhelms (1987) and Spudis (1993), of which there are 46 (see Table 1). These basins have been grouped into 15 relative age classes (with 1 being the youngest and 15 the oldest), and although absolute ages are known only for less than a handful of these, it is likely that they all formed between about 3.8 and 4.4 billion years ago (see Stöffler and Ryder, 2001). Two of these basins have uncertain relative ages (Cruger-Sirsalis and Milne), and for these we take the average of their estimated upper and lower age bounds. Furthermore, we neglect the oldest and largest basin on this list in our study (South Pole-Aitken) since this giant basin probably altered the moment's of inertia, and hence stable orientation, of the Moon. (It would be a remarkable coincidence if the largest impact basin on the Moon were to have formed at exactly $180^{\circ}$ longitude.)

Strom et al. (2005) have shown that the size-frequency distribution of objects colliding with the Moon was probably different when the ancient basins formed than afterwards. It would therefore be useful to analyze the size-frequency distribution of impact basins and to quantify how this might have evolved with time. Unfortunately, the small number of basins within the 15 relative age groups renders any statistical testing inconclusive. Furthermore, as discussed previously, the size of the transient cavity for only a few of these basins has been constrained by geophysical means, and these are often found to be inconsistent with the main rim diameters as determined by geomorphological analyses (see Table 1). For this reason, we are constrained to analyze only temporal variations in the spatial distribution of impact basins, irrespective of size.

To quantify the distribution of lunar impact basins, we use the statistic

$$
\overline{\mathbf{r}}=\frac{1}{N} \sum_{i=1}^{N} \hat{\mathbf{r}}_{i}
$$

where $N$ is the number of impact basins and $\hat{\mathbf{r}}_{i}$ is a unit vector pointing in the direction of the $i$ th basin. If the basin locations were randomly distributed, the expectation value of this statistic would be identically zero. The observed 
direction and magnitude of $\overline{\mathbf{r}}$ for a given set of basins are thus indicators of any spatial asymmetry that might have existed in the cratering rate. Given a particular number of basins, we determine the probability that a spatially uniform cratering rate could have given rise to the observed value of $\overline{\mathbf{r}}$ by performing Monte Carlo simulations. (We note that nearly all crater chronology studies of the Moon assume a spatially uniform cratering rate.)

[Fig. 2 about here.]

The upper image of Figure 2 shows the locations of the impact basins used in this study, and the lower image shows the direction of $\overline{\mathbf{r}}$ for all basins younger and contemporary to a given age group (blue), or older and contemporary to a given age group (red). As is seen, the younger basins (age groups $\leq 12$ ) are, as might be expected, preferentially located on the western hemisphere of the Moon. In contrast, when considering only those basins that are older or equal to a given age group, it is seen that the older basins are preferentially located on the eastern hemisphere of the Moon. The simplest hypothesis to explain this observation is that the youngest basins formed when the Moon was in its current orientation, but that the oldest basins formed when it was in an orientation rotated $180^{\circ}$ about its spin axis.

[Fig. 3 about here.]

The next question to address is whether the magnitudes of the observed asymmetries in basin locations (as quantified by the magnitude of $\overline{\mathbf{r}}$ ) are statistically significant, or if a uniform cratering rate could have given rise to these by chance. The upper panel of Figure 3 plots the observed magnitude of $\overline{\mathbf{r}}$ for all basins with ages either greater or less than a given age group, and the lower panel plots the corresponding probabilities that such values (or larger) could have occurred by chance. When considering all those basins younger and contemporary to a given age group (blue), the magnitude of $\overline{\mathbf{r}}$ is seen to be the largest for the youngest basins (age groups $\leq 4$, which comprise 10 basins). Nevertheless, the lower panel of Figure 3 shows that there is a high probability that these values could have occurred by chance. This statistical non-significance is likely to be a consequence of both the small number of young basins and the small value of the expected cratering asymmetry when these basins formed. Furthermore, it is also possible that the Moon could have been reoriented several times by the younger impact basins, giving rise to a more uniform time-integrated cratering rate.

When basins older and contemporary to a given age group are considered (red), the magnitude of $\overline{\mathbf{r}}$ is seen to be largest for the oldest basins, in contrast to the case of the youngest basins. Furthermore, there is less than a $5 \%$ probability (which are 2- $\sigma$ events) that the magnitude of $\overline{\mathbf{r}}$ could have occurred by chance for certain age groups. In particular, the probability that basins are randomly 
distributed for age groups older or equal to 11,12 , and 13 is $3.3,1.6$ and $1.3 \%$, respectively. These low probabilities for the oldest basins are likely to be a consequence of the greater number of basins in these groups, and might also indicate that the cratering asymmetry was larger in the past when the Moon was closer to the Earth.

[Fig. 4 about here.]

In addition to testing whether the lunar basins are randomly distributed or not, we next investigate whether the observed magnitude and direction of the observed asymmetry are consistent with having a higher cratering rate near either the Moon's apex or antapex of motion. In particular, for a given number of basins, we have used Monte Carlo simulations to determined the probability that a uniform cratering rate could have given rise to (1) a magnitude of $\overline{\mathbf{r}}$ being as great or greater than observed and (2) an angular distance between $\overline{\mathbf{r}}$ and either the Moon's apex or antapex of motion being as small or smaller than observed. For the case where basins younger and contemporary to a given age group are considered, no age groups are found that require a higher cratering rate directed towards the Moon's apex of motion (the only exception is age group 1, which contains one crater that is located almost exactly at the current apex of motion). In contrast, when considering basins that are older and contemporary to a given age group, certain age groups are found to be statistically significant at the $3-\sigma$ level. In particular, there is less than a $0.27 \%$ probability that the observed magnitude of $\overline{\mathbf{r}}$ and angular distance between $\overline{\mathbf{r}}$ and the Moon's antapex of motion could have occurred by chance for age groups greater or equal to 12 and 13. This result is consistent with the hypothesis that the oldest basins formed when the orientation of the Moon was rotated about its spin axis by $180^{\circ}$.

We have performed the same statistical tests for all possible contiguous ranges of relative age groups (instead of just greater or less than a certain age). Given the smaller number of basins in many of these tests, most age ranges are consistent with being derived from a uniform cratering rate. The only exceptions (at the $5 \%$ level) are the individual age groups 12 and 13, as well as the age range $5-12$. The asymmetry direction for the first two groups are on the Moon's eastern hemisphere (which is consistent with the above results), whereas the last range is located close to $180^{\circ}$ longitude (and which has an ambiguous interpretation). Furthermore, no additional age ranges were found to be non-random and directed towards the apex or antapex of motion at better than the 3- $\sigma$ level. Finally, we note that there appear to be more impact basins on the Moon's southern hemisphere than its northern hemisphere, and statistical tests show that this is significant at the $5 \%$ level when all age groups are considered. Nevertheless, if the oldest group is ignored (group 14), this north-south asymmetry is no longer statistically significant. 


\section{Discussion}

One fundamental uncertainty with this study concerns the completeness of the employed data set of impact basins. In particular, it is possible that all basins greater than a certain size might not yet have been discovered on the Moon. As an example, it is noted that the basin Cruger-Sirsalis, which is covered by Orientale ejecta, was not discovered until the acquisition of topographic data from the Clementine mission (Cook et al., 2002). In a similar manner, it is possible that the mare basalts on the near-side hemisphere might have obscured from view some of the older basins. While this second possibility can not be excluded, it is clear from the upper image in Figure 2 that the largest "deficit" of old basins (plotted in red) is not beneath the maria, but rather within the highlands (in particular, north-east of the South Pole-Aitken basin and close to both poles).

An additional problem with the basin data set is that the criteria used to rank the relative ages of basins might sometimes have given incorrect results. For instance, it is possible that criteria related to the degradation of surface morphology might in some cases be more a function of proximity to younger basins than relative age. Regardless, when considering basins whose ages are less than or greater than a given age group, we would not expect small errors in a few relative ages to dramatically alter our conclusions.

Barring problems with our employed data set in Table 1, the distribution of lunar impact basins seems to indicate at the 3- $\sigma$ level that the oldest basins formed preferentially near the Moon's current antapex of motion $\left(0^{\circ} \mathrm{N}, 90^{\circ}\right.$ E). If this is true, the simplest way to explain this observation is to hypothesize that the present "near side" of the Moon was once facing away from the Earth and that the present-day eastern hemisphere was subjected to a higher cratering rate at this time as a result of the Moon's synchronous rotation. A large impact event would then be required to unlock the Moon from synchronous rotation and the geographic locations of the apex and antapex of motion would have to have been switched during synchronization. It is natural to ask which lunar impact basin might have been responsible for this event.

Based on the results plotted in Figure 4, it is seen that basins with ages older and contemporary to group 13 have the highest probability of having formed when the Moon was rotated $180^{\circ}$ about its spin axis. Basins older or equal to age groups 11 and 12 possess nearly the same probability, whereas the probability that this distribution could have occurred by chance sharply increases when younger groups are included. The simplest possibility is that the required basin formed near the boundary of the groups 10-11, 11-12, or 1213. The inclusion of groups 10, 11, and 12 in our Monte Carlo simulations each increases the probability that the distribution of old basins could have occurred 
by chance, and these three groups comprise six craters: Lorentz, Smythii, Coulomb-Sarton, Keeler-Heaviside, Poincare, and Ingenii. Of these six basins, only the size of the Smythii basin has been constrained by geophysical means. With a diameter of $567 \mathrm{~km}$, the Smythii event would have been capable of reorienting the Moon under average impact conditions (see Figure 1), even if the Earth-Moon separation at this time were only 25 Earth radii. Indeed, given that the Smythii basin is located at the equator, this event would have maximized the change in the Moon's rotation rate about the spin axis with respect to one of the three impact geometry variables. We can not dismiss the possibility that one of the other five basins might have been responsible for this event (nor, perhaps, that it was one of the youngest basins within age group 13 itself), but Smythii's size, relative age and location single it out as one of the prime suspects. If the Smythii basin could be dated by absolute means, this would potentially date an important event in lunar history.

One question that we can ask about the magnitude of the observed cratering asymmetry is whether it is consistent with what would be expected from the present-day orbital distribution of near-Earth objects (NEOs). In particular, for basins older and contemporary to group 11 (of which there are 20), the magnitude of $\overline{\mathbf{r}}$ is seen to be about 0.4. Using the simulations of Le Feuvre and Wieczorek (2008) and Le Feuvre (2008) with the NEO model of Bottke et al. (2002), we find that the average value of $\overline{\mathbf{r}}$ when forming 20 basins should not be more than 0.27 , even if the Moon were separated from the Earth by only 5 Earth radii. Nevertheless, given the small number of basins in these simulations, the 1- $\sigma$ upper limit of this theoretical distribution can reach about 0.38 , suggesting that the observed cratering asymmetry could have been created by an orbital distribution of NEOs not too different from the present population. Regardless, we note that if the encounter velocities of the NEOs with the Earth-Moon system were smaller in the past than at the present time (such as might be the case for the "left overs" of accretion or destabilized Earth Trojans), the apex-antapex asymmetry could have been considerably larger. We also note that the size-frequency distributions of old craters in the lunar highlands differ from those of the youngest volcanic plains (Strom et al., 2005), and this might imply that the orbital elements of the NEO population was different in the ancient past.

Throughout most of this paper, we have assumed implicitly that the direction of the Moon's principal moments of inertia have not changed over time, and hence that there were only two stable geographic orientations of the Moon at the time of basin formation. However, as noted by Melosh (1975), large impact basins could alter the Moon's moments of inertia, perhaps giving rise to a short period of true polar wander as the Moon reoriented to achieve its minimum energy configuration (see also Runcorn, 1980, 1982). Since basin floors are now suspected to rebound to a near isostatic state on timescales that are much shorter than previously assumed (e.g., Melosh, 1989), basin-related 
true polar wander is probably not an important process, with the probable exception of the South Pole-Aitken basin. Nevertheless, it is possible that internal dynamics associated with mantle convection, and/or the formation and evolution of the Procellarum KREEP terrane (e.g., Jolliff et al., 2000; Shearer et al., 2006) (such as by the concentration of dense ilmenite phases in the mantle beneath this near-side province), could have altered the moments of inertia enough to have given rise to some amount of true polar wander. Figure 2 shows that the direction of the maximum cratering rate as implied by the oldest basins is somewhat offset from the Moon's current antapex of motion, and one could speculate that this might be a reflection of more than $30^{\circ}$ of true polar wander following the formation of these impact basins.

In addition to subjecting the current antapex of motion to a higher cratering rate in the past, a $180^{\circ}$ reorientation of the Moon could have potentially given rise to several other effects. First, as a result of the Earth's protective magnetosphere, the net quantity of solar-wind implanted volatiles is expected to be smaller on the near-side hemisphere of the Moon than on its far-side hemisphere. If one or more reorientation events occurred, this might be visible in the abundances of solar-wind implanted volatiles found in ancient buried regolith horizons. Second, as the Moon despun to synchronous rotation, energy would have been dissipated at the core-mantle boundary if the lunar core was molten (see Williams et al., 2001). Conceivably, this could have stopped a geodynamo if one was operating at this time (at least temporarily), or perhaps might even have been capable of starting a short-lived nutationally driven dynamo. Third, as the solid body of the Moon rotated though the Earth's tidal potential, stresses would have been set up in the lithosphere, and this could have led conceivably to a global fracture system (cf., Melosh, 1980; Helfenstein and Parmentier, 1985).

Finally, we note that in addition to unlocking the Moon from synchronous rotation, some large impact events might have been capable of placing the Moon (at least temporarily) into a higher-order spin-orbit resonance. Alternatively, such an impact could have destabilized a higher-order resonant spin state that might have existed early in the Moon's orbital evolution. The triaxial shape of the Moon is much more flattened than expected for the current Earth-Moon separation, and many authors have speculated that this might represent an equilibrium shape that was frozen into the lithosphere when the Moon was rotating synchronously and closer to the Earth (e.g., Lambeck and Pullan, 1980). Garrick-Bethell et al. (2006) have noted that the triaxial shape of the Moon could also be explained as being an equilibrium shape if the Moon were locked into an eccentric 3/2 spin-orbit resonance with an Earth-Moon separation of about 25 Earth radii. An unresolved problem with this latter hypothesis is the manner in which the Moon could have acquired a spin rate greater than synchronous, and how this resonance was ultimately destabilized. Here we note that one of these phenomena could have been achieved by the 
impact that formed the South Pole-Aiken basin. To show this, we first calculated the bolide diameters and velocities required to spin the Moon up from synchronous rotation to that of the $3 / 2$ resonance (or vice versus) when the Earth-Moon separation was 25 Earth radii. Inserting these values into crater scaling laws in the gravity regime, and assuming an average impact geometry, we find that the resulting crater diameter would have to lie between about 850 and $1450 \mathrm{~km}$ for impact velocities of 5 and $50 \mathrm{~km} \mathrm{~s}^{-1}$, respectively. Since the diameter of the South Pole-Aitken basin is in excess of $2000 \mathrm{~km}$, this basin could have easily been the cause of such a short-lived $3 / 2$ resonant spin rate, or the manner in which such a previously existing higher-order resonant spin state was destabilized.

\section{Conclusions}

As a consequence of synchronous rotation, more impact events should occur near the Moon's apex of motion on its western hemisphere than its antapex of motion on its eastern hemisphere. Impact events that formed the largest lunar basins could have temporarily unlocked the Moon from synchronous rotation, and following the re-establishment of this resonant spin state, there would be a 50/50 chance for the previous "near side" of the Moon to be directed away from the Earth. If this were to have occurred, basins that formed during certain time intervals would be expected to cluster near the current antapex of motion on the eastern hemisphere of the Moon.

The spatial distribution of known lunar impact basins implies the following:

(1) The youngest basins (relative age groups $\leq 12$ ) are preferentially located on the western hemisphere of the Moon, close to the Moon's current apex of motion. Nevertheless, if the cratering rate were uniform across the lunar surface, there would be more than a $5 \%$ probability that this configuration could have occurred by chance.

(2) The oldest lunar basins are not consistent with having formed from a uniform cratering rate. For the basins that are older and contemporary to age groups 11,12 , and 13 , there is only a $3.3,1.6$ and $1.3 \%$ probability, respectively, that the magnitude of the observed asymmetry could have occurred by chance.

(3) The oldest basins are preferentially located on the eastern hemisphere of the Moon, close to the Moon's current antapex of motion. If the lunar cratering rate were spatially uniform, there would be less than a $0.27 \%$ probability that the magnitude of the cratering asymmetry would be as large as observed, and that the direction of this asymmetry would be as close as observed to the Moon's antapex of motion for age groups older and contemporary to 12 and 13 . 
The simplest interpretation of the above observations is that the oldest lunar impact basins formed when the present-day "near side" of the Moon was directed away from the Earth and that a single impact subsequently reoriented the Moon about its spin axis by $180^{\circ}$. Such a reorientation most probably occurred during one of the age groups 10,11 or 12 , which comprise six impact basins. Of these six, only the size of Smythii has been constrained by geophysical means, and this basin would have been sufficient to have unlocked the Moon from synchronous motion. If this basin could be dated by absolute means, this would date potentially an important event in lunar history.

The main problem with this analysis and the above conclusions concerns the fidelity of the employed data set of lunar impact basins and the ranking of these basins in terms of relative age groups. Given that there are only 46 known impact basins, the discovery of additional basins using improved topographic maps from ongoing and upcoming missions (such as Kaguya, Chang'e1, Chandrayaan-1, and Lunar Reconnaissance Orbiter) would benefit enormously the statistical tests applied in this study. We note that a preliminary analysis of Clementine topographic data sets by Frey (2008b) has identified 47 additional potential basins, and that it should be possible to place these into a relative age chronology (see, for example, Wilhelms, 1987; Frey, 2008a; Werner, 2008). Even if only a handful of these potential basins are confirmed to be legitimate impact basins, these would aid tremendously in testing the hypothesis that one or more impact events led to a $180^{\circ}$ reorientation of the Moon.

In addition to the acquisition of improved topographic data sets over the next few years, our knowledge of the Moon's gravity field will be dramatically improved from analyses of data obtained from the ongoing Kaguya mission, as well as the mission GRAIL (Gravity Recovery and Interior Laboratory) that is slated for a launch in 2011. From these data products, it will be possible to reconstruct the size of many impact basins that are located on the far-side hemisphere of the Moon, from which it will be possible to determine which of these would have been capable of reorienting the Moon. In the more distant future, the hypothesis that one or more reorientations of the Moon occurred could be tested by measuring the abundance of solar wind implanted volatiles in ancient regolith horizons.

\section{Acknowledgements}

We thank S. Werner, H. J. Melosh, P. Pinet, and an anonymous reviewer for constructive reviews and comments that improved this manuscript. All figures were created using the Generic Mapping Tools of Wessel and Smith (1991). This is IPGP contribution 2456. 


\section{References}

Ahrens, T. J., Harris, A. W., 1994. Deflection and fragmentation of NEAs. In: Gehrels, T. (Ed.), Hazards due to comets and asteroids. Univ. of Arizona Press, Tucson, pp. 897-928.

Bottke, W., Love, S. G., Tytell, D., Glotch, T., 2000. Interpreting the elliptical crater populations on Mars, Venus, and the Moon. Icarus 145, 108-121.

Bottke, W., Morbidelli, A., Jedicke, R., Petit, J.-M., Levison, H. F., Michel, P., Metcalfe, T. S., 2002. Debiassed orbital and absolute magnitude distribution of the near-Earth objects. Icarus 156, 399-433.

Burns, J. A., 1986. The evolution of satellite orbits. In: Burns, J. A., Mathews, M. S. (Eds.), Satellites. Univ. Arizona Press, Tucson, pp. 117-158.

Cook, A. C., Spudis, P. D., Robinson, M. S., Watters, T. R., 2002. Lunar topography and basins mapped using a Clementine stereo digital elevation model. Lunar Planet. Sci. 33, Abstract 1281.

Croft, S. K., 1985. The scaling of complex craters. Proc. Lunar Planet. Sci. Conf, 15, J. Geophys. Res., supp. 90, C828-C842.

Frey, H., 2008a. Ages of very large impact basins on Mars: Implications for the late heavy bombardment in the inner solar system. Geophys. Res. Lett. 35, L13203.

Frey, H. V., 2008b. Previously unrecognized large lunar impact basins revealed by topographic data. Lunar Planet. Sci. 29, abstract 1344.

Gallant, J., Gladman, B., Ćuk, M., 2006. Current bombardment of the EarthMoon system: Emphasis on cratering asymmetries. arXiv Astrophysics eprint astro-ph/0608373v1.

Garrick-Bethell, I., Wisdom, J., Zuber, M. T., 2006. Evidence for a past higheccentricity lunar orbit. Science 313, 652-655.

Goldreich, P., Peale, S., 1966. Spin-orbit coupling in the Solar System. Astron. J. 71, 425-438.

Helfenstein, P., Parmentier, E. M., 1985. Patterns of fracture and tidal stresses due to nonsynchronous rotation: implications for fracturing on Europa. Icarus $61,175-184$.

Herrick, R. R., Forsberg-Taylor, N. K., 2003. The shape and appearance of craters formed by oblique impact on the Moon and Venus. Meteorit. Planet. Sci. 38, 1551-1578.

Hikida, H., Wieczorek, M. A., 2007. Crustal thickness of the Moon: New constraints from gravity inversions using polyhedral shape models. Icarus 192, 150-166.

Holsapple, K. A., 1993. The scaling of impact processes in planetary science. Annu. Rev. Earth Planet. Sci. 21, 333-373.

Holsapple, K. A., 2004. About deflecting asteroids and comets. In: Belton, M. J. S., Morgan, T. H., Samarasinha, N. H., Yeomans, D. K. (Eds.), Mitigation of hazardous comets and asteroids. Cambridge Univ. Press, pp. 113-140.

Holsapple, K. A., Housen, K. R., 2007. A crater and its ejecta: An interpretation of Deep Impact. Icarus 187, 345-356. 
Horedt, G. P., Neukum, G., 1984. Cratering rate over the surface of a synchronous satellite. Icarus 60, 710-717.

Johnson, J. R., Swindle, T. D., Lucey, P. G., 1999. Estimated solar windimpanted Helium-3 distribution on the Moon. Geophys. Res. Lett. 26, 385388.

Jolliff, B. J., Gillis, J. J., Haskin, L. A., Korotev, R. L., Wieczorek, M. A., 2000. Major lunar crustal terranes: Surface expressions and crust-mantle origins. J. Geophys. Res. 105, 4197-4216.

Lambeck, K., Pullan, S., 1980. The lunar fossil bulge hypothesis revisited. Phys. Earth Planet. Inter. 22, 29-35.

Le Feuvre, M., 2008. Modélisation de la formation des cratères d'impact à la surface des planètes et des satellites (in french). Ph.D. thesis, Univ. Paris VII.

Le Feuvre, M., Wieczorek, M. A., 2008. Nonuniform cratering of the terrestrial planets. Icarus 197, 291-306.

Lissauer, J. J., 1985. Can cometary bombardment disrupt synchronous rotation of planetary satellites? J. Geophys. Res. 90, 11289-11293.

Melosh, H. J., 1975. Large impact craters and the Moon's orientation. Earth Planet. Sci. Lett. 26, 353-360.

Melosh, H. J., 1980. Tectonics on a tidally distorted planet. Icarus 43, 334-337.

Melosh, H. J., 1989. Impact Cratering: A Geologic Process. Oxford Univ. Press, New York.

Melosh, H. J., Nemchinov, I. V., Zetzer, Y. I., 1994. Non-nuclear strategies for deflecting comets and asteroids. In: Gehrels, T. (Ed.), Hazards due to comets and asteroids. Univ. of Arizona Press, Tucson, pp. 1111-1134.

Morota, T., Furumoto, M., 2003. Asymmetrical distribution of rayed craters on the Moon. Earth Planet. Sci. Lett. 206, 315-323.

Morota, T., Ukai, T., Furumoto, M., 2005. Influence of the asymmetrical cratering rate on the lunar cratering chronology. Icarus 173, 322-324.

Peale, S. J., 1975. Dynamical consequences of meteorite impacts on the moon. J. Geophys. Res. 80, 4939-4946.

Pinet, P., 1985. Lunar impact flux distribution and global asymmetry revisited. Astron. Astrophys. 151, 222-234.

Ross, M. N., Schubert, G., 1989. Evolution of the lunar orbit with temperatureand frequency-dependent dissipation. J. Geophys. Res. 94, 9533-9544.

Runcorn, S. K., 1980. Lunar polar wandering. Proc. Lunar Planet. Sci. Conf. 11, 1867-1877.

Runcorn, S. K., 1982. Primeval displacements of the lunar pole. Phys. Earth Planet. Int. 29, 135-147.

Shearer, C. K., Hess, P. C., Wieczorek, M. A., Pritchard, M. E., Parmentier, M. E., Borg, L. E., Longhi, J., Elkins-Tanton, L. T., Neal, C. R., Antonenko, I., Canup, R. M., Halliday, A. N., Grove, T. L., Hager, B. H., Lee, D.C., Wiechert, U., 2006. Thermal and magmatic evolution of the Moon. In: Jolliff, B. J., Wieczorek, M. A., Shearer, C. K., Neal, C. R. (Eds.), New views of the Moon. Vol. 60 of Rev. Min. Geochem. Mineral. Soc. Amer., pp. 
$365-518$.

Shoemaker, E. M., Wolf, R. F., 1982. Cratering time scales for the Galilean satellites. In: Morison, D. (Ed.), Satellites of Jupiter. Univ. Arizona Press, Tucson, pp. 277-339.

Spudis, P. D., 1993. The geology of multi-ring impact basins. Cambridge Univ. Press, New York.

Stöffler, D., Ryder, G., 2001. Stratigraphy and isotope ages of lunar geologic units: Chronological standard for the inner solar system. Space Sci. Rev. 96, 9-54.

Strom, R. G., Malhotra, R., Ito, T., Yoshida, F., Kring, D. A., 2005. The origin of planetary impactors in the inner Solar System. Science 309, 1847-1849.

Webb, D. J., 1982. Tides and the evolution of the Earth-Moon system. Geophys. J. R. Astron. Soc. 70, 261-271.

Werner, S. C., 2008. The early martian evolution-constraints from basin formation ages. Icarus 195, 45-60.

Wessel, P., Smith, W. H. F., 1991. Free software helps map and display data. EOS 72 (441).

Wieczorek, M., Phillips, R. J., 1999. Lunar multiring basins and the cratering process. Icarus 139, 246-259.

Wilhelms, D. E., 1987. The geologic history of the Moon. Vol. 1348. U.S. Geol. Surv. Spec. Pap.

Williams, G. E., 2000. Geologic constraints on the Precambrian history of Earth's rotation and the Moon's orbit. Rev. Geophys. 38, 37-59.

Williams, G. E., 2004. Earth's Precambrian rotation and the evolving lunar orbit: Implications of tidal rhythmite data for paleogeophysics. In: Eriksson, P. G., Altermann, W., Nelson, D. R., Mueller, W. U., Catuneanu, O. (Eds.), The Precambrian Earth: Tempos and events, Developments in Precambrian geology, 12. Elsevier, Amsterdam, pp. 473-484.

Williams, J. G., Boggs, D. H., Yoder, C. F., Ratcliff, J. T., Dickey, J. O., 2001. Lunar rotational dissipation in solid body and molten core. J. Geophys. Res. 106, 27933-27968.

Wood, J. A., 1973. Bombardment as a cause of the lunar asymmetry. Moon 8, 73-103.

Zahnle, K., Schenk, P., Sobieszczyk, S., Dones, L., Levison, H. F., 2001. Differential cratering of synchronously rotating satellites by ecliptic comets. Icarus 153, 111-129. 

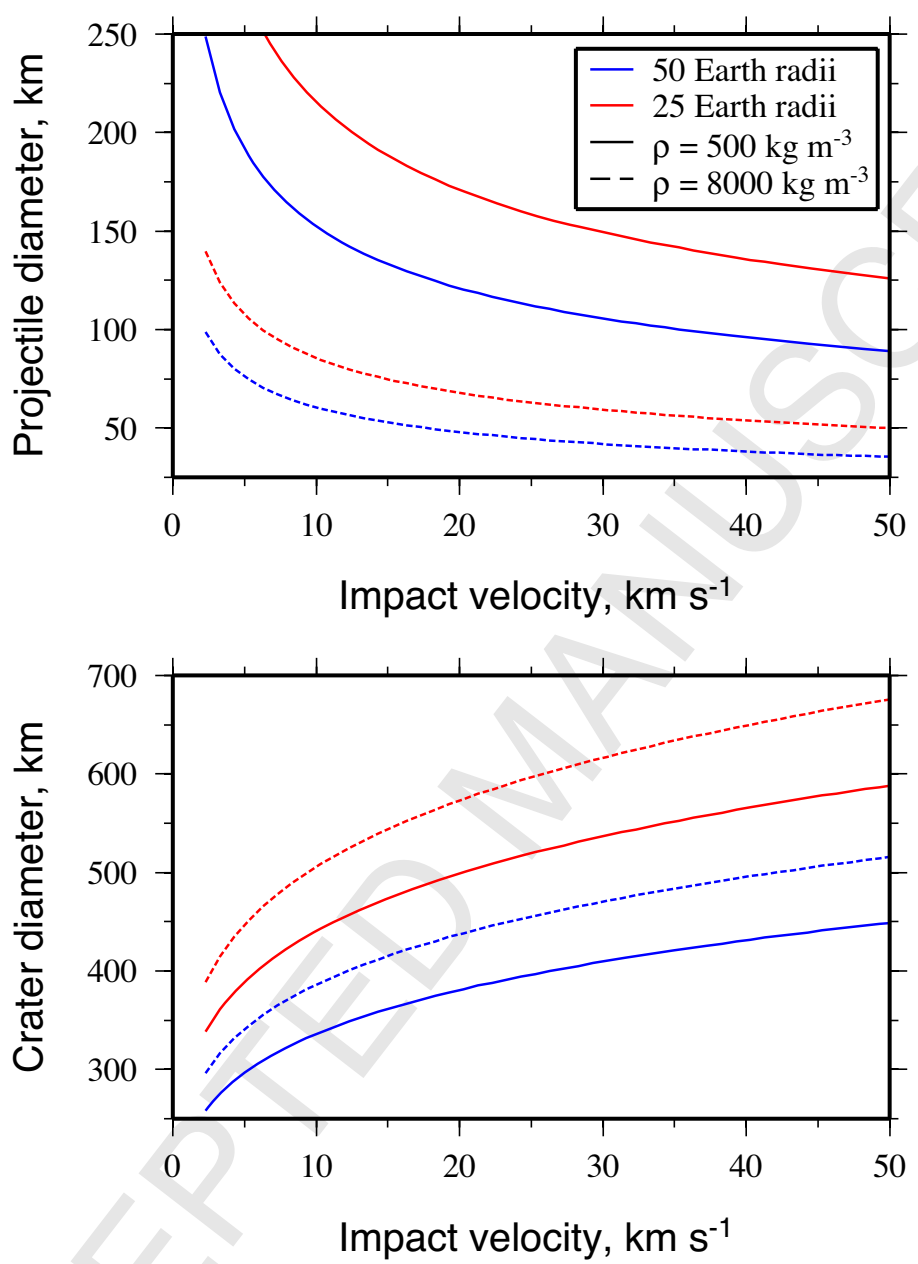

Fig. 1. Minimum projectile diameter (top) and minimum crater diameter (bottom) required to unlock the Moon from synchronous rotation as a function of impact velocity for the case of an average impact geometry. 

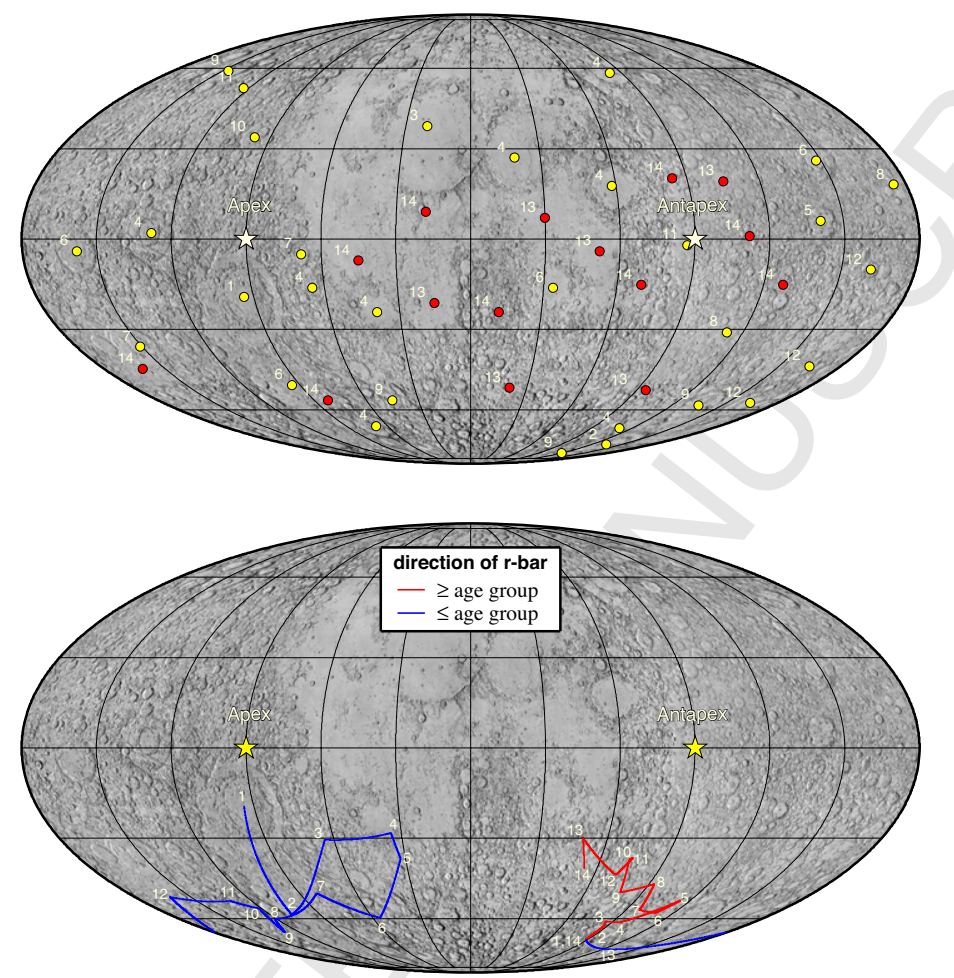

Fig. 2. (top) Locations of lunar impact basins in Table 1 (excluding South Pole-Aitken) with numbers indicating the relative age group (age group 1 is the youngest and age group 14 is the oldest). Basins younger and contemporary to group 11 are plotted in yellow, whereas older basins are plotted in red. (bottom) Position on the lunar surface of the vector $\overline{\mathbf{r}}$ for all basins younger and contemporary to (blue), or older and contemporary to (red), a given age group. Both images are in a Molleweide projection with a central meridian of $0^{\circ}$. 

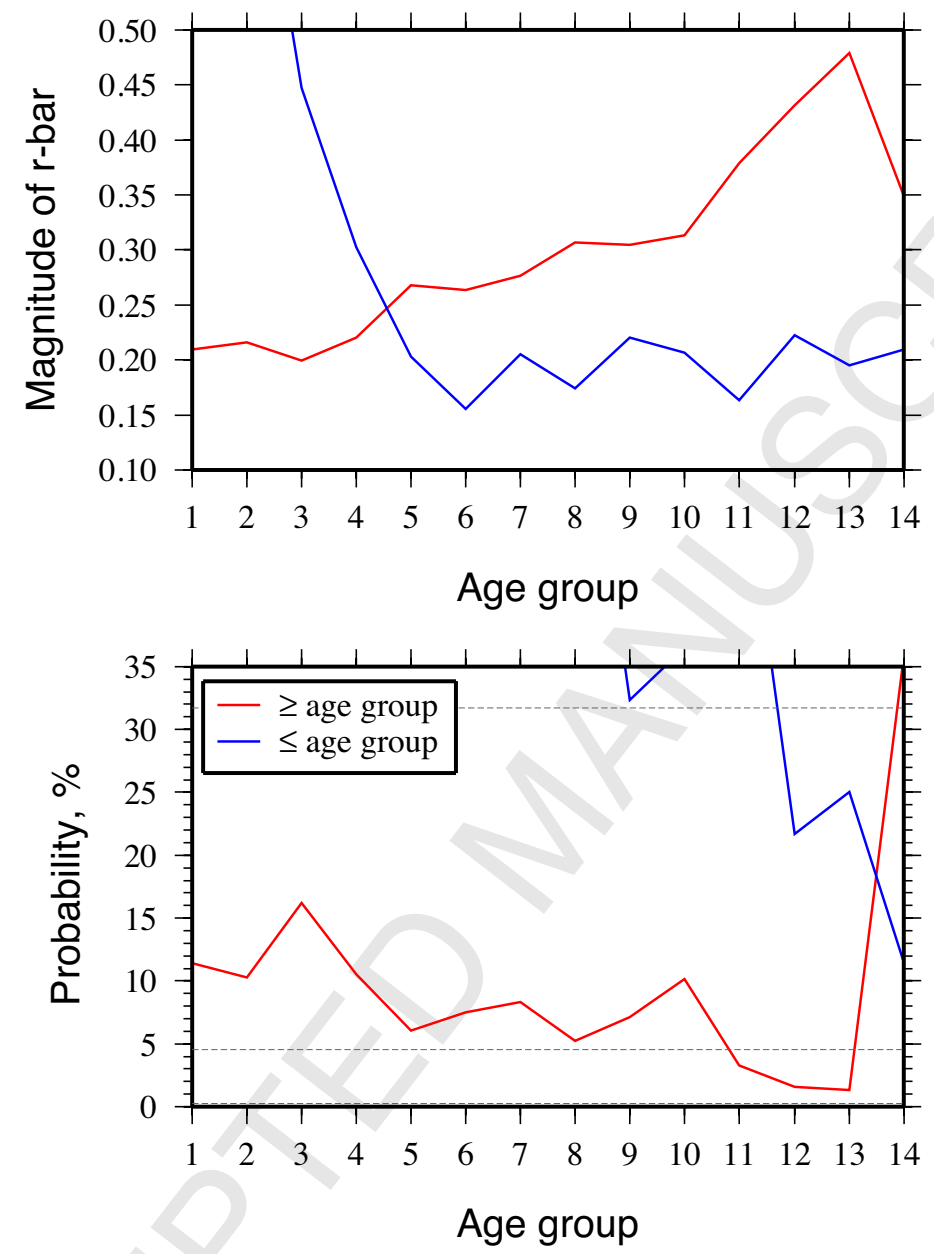

Fig. 3. (top) Magnitude of $\overline{\mathbf{r}}$ for all basins younger and contemporary to a given age group (blue) and older and contemporary to a given age group (red). (bottom) Probability that the above values of $\overline{\mathbf{r}}$ could occur by chance for a spatially uniform cratering rate. Horizontal lines denote 1- and 2- $\sigma$ events (i.e., probabilities of 31.8 and $4.6 \%$, respectively). Age group 1 is the youngest, and age group 14 is the oldest. 


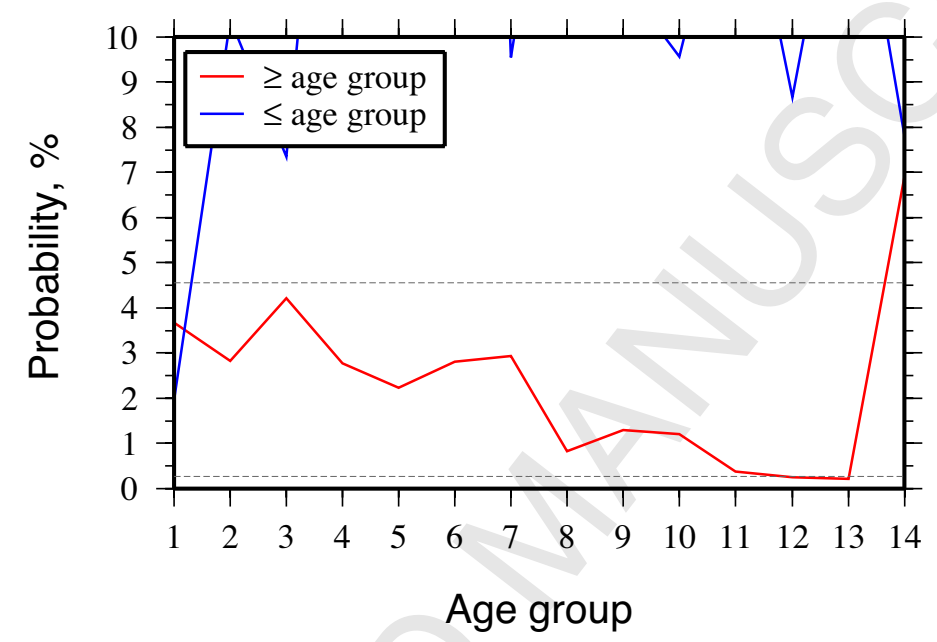

Fig. 4. Probability that the magnitude of $\overline{\mathbf{r}}$ could occur by chance and be as close as observed to the Moon's apex of motion for all basins younger and contemporary to a given age group (blue). Probability that the magnitude of $\overline{\mathbf{r}}$ could occur by chance and be as close as observed to the Moon's antapex of motion for all basins older and contemporary to a given age group (red). Horizontal lines denote 2- and $3-\sigma$ events (i.e., probabilities of 4.6 and $0.27 \%$, respectively). 
Table 1

Lunar impact basins.

\begin{tabular}{|c|c|c|c|c|c|}
\hline Basin name & (latitude, longitude) $^{*}$ & Relative age $^{\dagger}$ & Geologic period $^{\ddagger}$ & Main rim diameter ${ }^{\S}, \mathrm{km}$ & Excavation cavity diameter ${ }^{\star}, \mathrm{km}$ \\
\hline Orientale & $\left(-19^{\circ},-94^{\circ}\right)$ & 1 & I & 930 & 383 \\
\hline Schrodinger & $\left(-76^{\circ}, 134^{\circ}\right)$ & 2 & I & 320 & \\
\hline Imbrium & $\left(38^{\circ},-20^{\circ}\right)$ & 3 & I & 1160 & 895 \\
\hline Sikorsky-Rittenhouse & $\left(-68^{\circ}, 111^{\circ}\right)$ & 4 & $\mathrm{~N}$ & 310 & \\
\hline Bailly & $\left(-67^{\circ},-68^{\circ}\right)$ & 4 & $\mathrm{~N}$ & 300 & \\
\hline Hertzsprung & $\left(2^{\circ},-128^{\circ}\right)$ & 4 & $\mathrm{~N}$ & 570 & \\
\hline Serenitatis & $\left(27^{\circ}, 19^{\circ}\right)$ & 4 & $\mathrm{~N}$ & 920 & 718 \\
\hline Crisium & $\left(17.5^{\circ}, 58.5^{\circ}\right)$ & 4 & $\mathrm{~N}$ & 740 & 560 \\
\hline Humorum & $\left(-24^{\circ},-39.5^{\circ}\right)$ & 4 & $\mathrm{~N}$ & 425 & 382 \\
\hline Humboldtianum & $\left(58^{\circ}, 83^{\circ}\right)$ & 4 & $\mathrm{~N}$ & 650 & 394 \\
\hline Mendeleev & $\left(6^{\circ}, 141^{\circ}\right)$ & 5 & $\mathrm{~N}$ & 365 & \\
\hline Mendel-Rydberg & $\left(-50^{\circ},-94^{\circ}\right)$ & 6 & $\mathrm{~N}$ & 420 & 337 \\
\hline Korolev & $\left(-4^{\circ},-158^{\circ}\right)$ & 6 & $\mathrm{~N}$ & 440 & \\
\hline Moscoviense & $\left(26^{\circ}, 148^{\circ}\right)$ & 6 & $\mathrm{~N}$ & 420 & \\
\hline Nectaris & $\left(-16^{\circ}, 34^{\circ}\right)$ & 6 & $\mathrm{~N}$ & 860 & 455 \\
\hline Apollo & $\left(-36^{\circ},-151^{\circ}\right)$ & 7 & $\mathrm{pN}$ & 480 & \\
\hline Cruger-Sirsalis & $\left(-16^{\circ},-65^{\circ}\right)$ & $1-7(4)$ & $\mathrm{I}-\mathrm{pN}$ & & 306 \\
\hline Grimaldi & $\left(-5^{\circ},-68^{\circ}\right)$ & 7 & $\mathrm{pN}$ & 440 & 206 \\
\hline Freundlich-Sharonov & $\left(18^{\circ}, 175^{\circ}\right)$ & 8 & $\mathrm{pN}$ & 600 & \\
\hline Milne & $\left(-31^{\circ}, 113^{\circ}\right)$ & $7-9(8)$ & $\mathrm{pN}$ & $262^{\sharp}$ & \\
\hline Birkhoff & $\left(59^{\circ},-147^{\circ}\right)$ & 9 & $\mathrm{pN}$ & 325 & \\
\hline Planck & $\left(-58^{\circ}, 136^{\circ}\right)$ & 9 & $\mathrm{pN}$ & 325 & \\
\hline Schiller-Zucchius & $\left(-56^{\circ},-45^{\circ}\right)$ & 9 & $\mathrm{pN}$ & 335 & \\
\hline Amundsen-Ganswindt & $\left(-81^{\circ}, 120^{\circ}\right)$ & 9 & $\mathrm{pN}$ & 335 & \\
\hline Lorentz & $\left(34^{\circ},-97^{\circ}\right)$ & 10 & $\mathrm{pN}$ & 365 & \\
\hline Smythii & $\left(-2^{\circ}, 87^{\circ}\right)$ & 11 & $\mathrm{pN}$ & 740 & 567 \\
\hline Coulomb-Sarton & $\left(52^{\circ},-123^{\circ}\right)$ & 11 & $\mathrm{pN}$ & 440 & \\
\hline Keeler-Heaviside & $\left(-10^{\circ}, 162^{\circ}\right)$ & 12 & $\mathrm{pN}$ & 500 & \\
\hline Poincare & $\left(-57^{\circ}, 164^{\circ}\right)$ & 12 & $\mathrm{pN}$ & 325 & \\
\hline Ingenii & $\left(-43^{\circ}, 165^{\circ}\right)$ & 12 & $\mathrm{pN}$ & 315 & \\
\hline Lomonosov-Fleming & $\left(19^{\circ}, 105^{\circ}\right)$ & 13 & $\mathrm{pN}$ & 620 & \\
\hline Nubium & $\left(-21^{\circ},-15^{\circ}\right)$ & 13 & $\mathrm{pN}$ & 690 & \\
\hline Fecunditatis & $\left(-4^{\circ}, 52^{\circ}\right)$ & 13 & $\mathrm{pN}$ & 690 & \\
\hline Mutus-Vlacq & $\left(-51^{\circ}, 21^{\circ}\right)$ & 13 & $\mathrm{pN}$ & 690 & \\
\hline Tranquilitatis & $\left(7^{\circ}, 30^{\circ}\right)$ & 13 & $\mathrm{pN}$ & 700 & \\
\hline Australe & $\left(-52^{\circ}, 95^{\circ}\right)$ & 13 & $\mathrm{pN}$ & 880 & \\
\hline Al-Khwarizmi-King & $\left(1^{\circ}, 112^{\circ}\right)$ & 14 & $\mathrm{pN}$ & 590 & \\
\hline Pingre-Hausen & $\left(-56^{\circ},-82^{\circ}\right)$ & 14 & $\mathrm{pN}$ & $300^{\#}$ & \\
\hline Werner-Airy & $\left(-24^{\circ}, 12^{\circ}\right)$ & 14 & $\mathrm{pN}$ & 500 & \\
\hline Balmer-Kapteyn & $\left(-15^{\circ}, 70^{\circ}\right)$ & 14 & $\mathrm{pN}$ & 500 & \\
\hline Flamsteed-Billy & $\left(-7^{\circ},-45^{\circ}\right)$ & 14 & $\mathrm{pN}$ & 570 & \\
\hline Marginis & $\left(20^{\circ}, 84^{\circ}\right)$ & 14 & $\mathrm{pN}$ & 580 & \\
\hline Insularum & $\left(9^{\circ},-18^{\circ}\right)$ & 14 & $\mathrm{pN}$ & 600 & \\
\hline Grissom-White & $\left(-44^{\circ},-161^{\circ}\right)$ & 14 & $\mathrm{pN}$ & $600^{\sharp}$ & \\
\hline Tsiolkovsky-Stark & $\left(-15^{\circ}, 128^{\circ}\right)$ & 14 & $\mathrm{pN}$ & 700 & \\
\hline South Pole-Aitken & $\left(-56^{\circ}, 180^{\circ}\right)$ & 15 & $\mathrm{pN}$ & 2600 & $2099^{b}$ \\
\hline \multicolumn{6}{|c|}{ 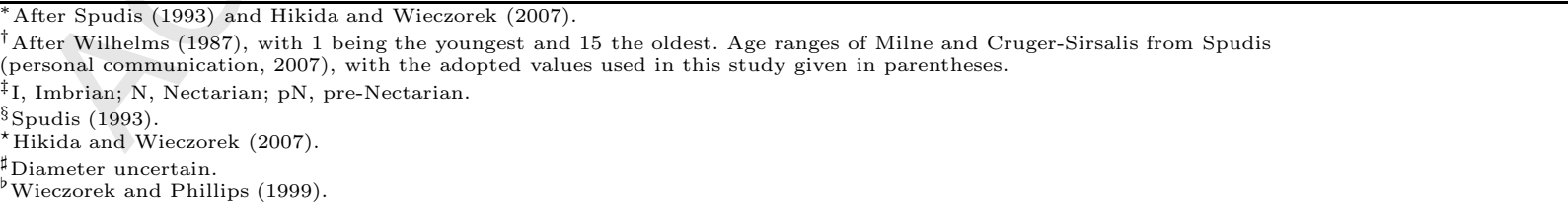 } \\
\hline
\end{tabular}

